\title{
Das Geburtsgewicht steuert beim Start ins Leben kräftig mit
}

\begin{abstract}
Fragestellung: Definiert das Geburtsgewicht eine viszerale Adipositas später im Leben?
\end{abstract}

\begin{abstract}
Hintergrund: Es gibt einen direkten pathophysiologischen Zusammenhang zwischen der Menge an innerem Bauchfett (viszerale Adipositas) und dem Diabetesrisiko sowie nahezu allen anderen chronischen Erkrankungen. Es wird zunehmend diskutiert, dass das intrauterine Umfeld in Form eines „embryonal programming“" sehr viele Rahmenbedingungen setzt, die im späteren Leben, auch Dekaden später, das Risiko für chronische Krankheiten steigern oder senken können. Allseits akzeptiert ist, dass ein höheres Geburtsgewicht mit einem höheren Diabetesrisiko assoziiert ist. Einige Studien lieferten ähnliche Information bei niedrigem Geburtsgewicht. Vieles davon wurde aber anekdotenhaft berichtet. Die aktuelle Studie hat diese Frage systematisch untersucht und analysiert, welcher Zusammen-

Originalie

Stansfield BK, Fain ME, Bhatia J et al. Nonlinear Relationship between Birth Weight and Visceral Fat in Adolescents. J Pediatr. 2016 Jul;174:185-92.

hang zwischen Geburtsgewicht und der Verteilung von viszeralem Fett und Biomarkern existiert, die für Typ2-Diabetes und ein erhöhtes kardiovaskuläres Risiko im späteren Leben stehen.
\end{abstract}

\section{- Kommentar von Prof. Dr. med. Peter Schwarz}

\section{Frühes Fett (be-)lastet oft lebenslang}

Diese systematische Querschnittsstudie zeigt, dass zu niedriges, aber auch zu hohes Geburtsgewicht für

Kinder, die bei der $\mathrm{Ge}$ burt normalgewichtig waren, starten beim Stoffwechsel am besten.

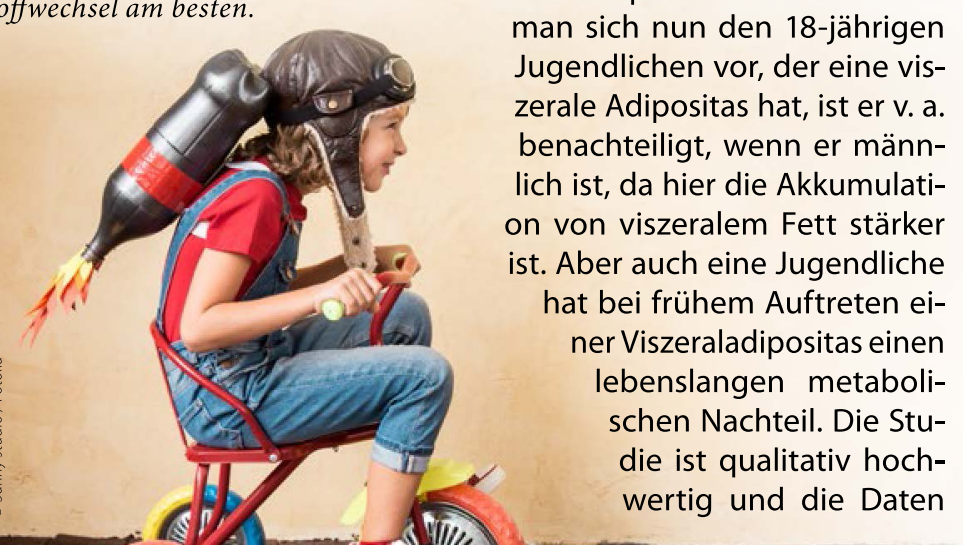

Patienten und Methoden: 575 Jugendliche im Alter zwischen 14 und 18 Jahren (52\% weiblich, $46 \%$ Schwarzafrikaner) kamen in die Studie. Das Geburtsgewicht wurde über Patientenakten und Geburtsdaten ermittelt.

Es wurden Nüchternzucker, Insulin, Lipide, Adiponektin, Leptin und CRP analysiert. Bei allen wurde die viszerale Fettmasse mit Magnetresonanzspektroskopie bestimmt.

Ergebnisse: In der Datenanalyse ergab sich eine U-förmige Kurve beim Zusammenhang von kardiometabolischen Risikofaktoren und Geburtsgewicht. Dabei wurden die Daten für Alter, Geschlecht, Rasse, Tanner-Stadium, körperliche Aktivität, sozioökonomischer Status und Body Mass Index adjustiert.

Weiterhin war der Zusammenhang signifikant für Insulinresistenz, Leptin und viszerales Fett. Interessanterweise ergab sich keine Assoziation für Nüchternglukose, Blutdruck, Gesamtcholesterin, LDL-Cholesterin, HDL-Cholesterin, Adiponektin und CRP sowie subkutane Fettmasse in Abhängigkeit vom Geburtsgewicht.

Schlussfolgerungen: Sowohl ein im Vergleich zum Durchschnitt niedriges als auch hohes Geburtsgewicht ist mit verstärktem Auftreten einer viszeralen Adipositas und kardiometabolischer Risiko-Biomarker assoziiert. wurden im Kontext einer Vielzahl von Einflussfaktoren analysiert. Was lernen wir? Wie vermutet ist eben nur ein normalgewichtes Baby normal und ein zu niedriges wie zu hohes Geburtsgewicht riskant. Interessanterweise besteht der Zusammenhang in erster Linie zur Akkumulation von viszeralem Fett, da hier vermutlich durch embryonales Programming das viszerale Fett als Speicherorgan stärker aktiviert ist. Bei den untersuchten Jugendlichen gab es keinen Zusammenhang mit dem Glukosestoffwechsel. Es ist aber für alle klar, dass Jugendliche mit viszeraler Adipositas und schon erhöhter Insulinresistenz auch ein erhöhtes Diabetesrisiko haben. Allerdings ist der Zuckerstoffwechsel hier sekundär und folgt dem Adipositasrisiko. Die Quintessenz dieser Studie ist daher: Normalgewicht ist fürs Baby der optimale Start ins Leben.

Prof. Dr. med. Peter Schwarz, MBA

Universität Dresden

peter.schwarz@uniklinikum-dresden.de 